Article

\title{
Optimization Design of Centrifugal Pump Flow Control System Based on Adaptive Control
}

\author{
Yuqin Wang $* \mathbb{\infty}$, Haodong Zhang, Zhibo Han and Xiaoqiang Ni \\ School of Mechanical Engineering, Chaohu University, Chaohu 238000, China; zhd2108956900@163.com (H.Z.); \\ hy269392@163.com (Z.H.); NXQ2534775625@163.com (X.N.) \\ * Correspondence: wyq111@126.com; +86-182-5655-6898
}

Citation: Wang, Y.; Zhang, H.; Han, $\mathrm{Z}$.; Ni, X. Optimization Design of Centrifugal Pump Flow Control System Based on Adaptive Control. Processes 2021, 9, 1538. https:// doi.org/10.3390/pr9091538

Academic Editor: Arkadiusz Gola

Received: 31 July 2021

Accepted: 26 August 2021

Published: 29 August 2021

Publisher's Note: MDPI stays neutral with regard to jurisdictional claims in published maps and institutional affiliations.

Copyright: (c) 2021 by the authors. Licensee MDPI, Basel, Switzerland. This article is an open access article distributed under the terms and conditions of the Creative Commons Attribution (CC BY) license (https:// creativecommons.org/licenses/by/ $4.0 /)$.

\begin{abstract}
In this paper, in order to improve the control characteristics of the centrifugal pump flow control system, a mathematical model of the centrifugal pump flow control system was established based on an analysis of the basic structures, such as the frequency converter, motor, and centrifugal pump. Based on the adaptive control theory, the recursive least squares algorithm with a forgetting factor was used to estimate the real-time parameters of the centrifugal pump control system, and the self-tuning PID control method was used to optimize the mathematical model of the centrifugal pump flow control system. The simulation results showed that the adjustment time of the optimized system was shortened by $16.58 \%$, and the maximum overshoot was reduced by $83.90 \%$, which improved the rapidity and stability of the transient response of the system. This showed that adaptive control had a significant effect on improving the robustness and anti-interference ability of the centrifugal pump control system. In order to further verify the accuracy of the self-tuning PID control method, a flow adaptive control system test platform was built. The test results showed that under the conditions of constant frequency and variable frequency, the actual flow rate of the centrifugal pump was always kept near the set flow rate, the error was small, and it had better real-time followability. The research results showed that adaptive control could revise the parameters in real-time according to changes to the centrifugal pump control system, which improved the stability and robustness of the system. Therefore, adaptive PID control could effectively improve the adaptability of centrifugal pumps to various complex working conditions and improve the working efficiency of centrifugal pumps.
\end{abstract}

Keywords: centrifugal pump; adaptive control; self-tuning PID; simulation analysis

\section{Introduction}

Pumps are widely used general machinery, mainly used in all aspects of production and life [1]. Centrifugal pumps account for a larger share of the total pump usage. There has been various research about centrifugal pumps recently. Gülich J. F et al. [2] predicted hydraulic design information, the turbine performance curve, torsional rotor vibration, partial load flow and hydraulic excitation force by studying the interaction between centrifugal pump fluid flow and vibration and material wear. Lobanoff VS et al. [3] accurately predicted the service life of the pump by studying the vibration and noise characteristics of the centrifugal pump, and performed rotor dynamics analysis and pump vibration diagnosis. L. Gevorkov, A et al. [4] proposed to simulate the flow control system of a centrifugal pump based on Simulink software, developed a specific centrifugal pump model, and carried out a series of simulations. With the advancement of science and technology and the development of intelligent control technology, research into automation control is also deepening, and the degree of industrial automation is also constantly improving. In industrial production, how to realize the automatic control of the output flow of centrifugal pumps is one of the important research directions of scientific and technological workers. Resolving this problem reasonably and effectively will save a lot of manpower and material resources and bring huge economic benefits. 
Centrifugal pump flow control refers to the regulation of the output flow of centrifugal pumps in industrial production, so that the output flow reaches a certain value set by the user. The output changes according to the rules set by the user to meet the normal use in industrial production. In practice, the external environments of centrifugal pumps are uncertain. The characteristics of the transmission medium will also be disturbed in the transmission process, resulting in the output flow of centrifugal pumps changing with a change in working conditions. At present, centrifugal pump flow control in industrial production is mostly regulated by manpower, which is inefficient and slow in response, which is not conducive to reducing production costs and improving economic benefits. Therefore, many scholars have studied the problem of centrifugal pump flow control. Sang-Ho Suh et al. [5] pointed out that the use of variable frequency regulation technology in flow control could reduce energy consumption to a certain extent and improve the service life of centrifugal pumps. Mingxin Chen et al. [6] used fuzzy PID control to adjust a centrifugal pump through frequency conversion, which improved the robustness of the flow control system, reduced overshoot, shortened the adjustment time and enhanced the anti-interference capability. Lu Jun et al. [7] used the $\mathrm{H} \infty$ method to control a centrifugal pump, so that the centrifugal pump flow rate could change in real-time with the external environment, and the following error was small.

In the current industrial control theory, the adaptive control theory has the advantages of eliminating the uncertainty of the controlled object, having good robustness to disturbance and being suitable for frequently changing working conditions. In this paper, the adaptive control theory is proposed to optimize the centrifugal pump flow control system. It can make the centrifugal pump flow control system more adaptable to frequently changing working conditions. This will reduce the influence of the environment on the output flow and improve work efficiency.

\section{Modeling of Centrifugal Pump Flow Control System}

\subsection{Structure Design}

The centrifugal pump flow control system consists of a centrifugal pump, controller, frequency converter, motor and flowmeter, as shown in Figure 1. When the centrifugal pump works, the flowmeter monitors the centrifugal pump output flow in real-time and feeds it back to the controller. The controller compares it with the set flow value. The motor input current frequency can be changed by controlling the inverter. When the motor speed changes, the output flow of the centrifugal pump can be adjusted. Through the real-time monitoring of the output flow and the real-time regulation of the motor, the automatic speed regulation of the centrifugal pump impeller is realized, and the automatic regulation control of the centrifugal pump output flow is completed $[8,9]$.

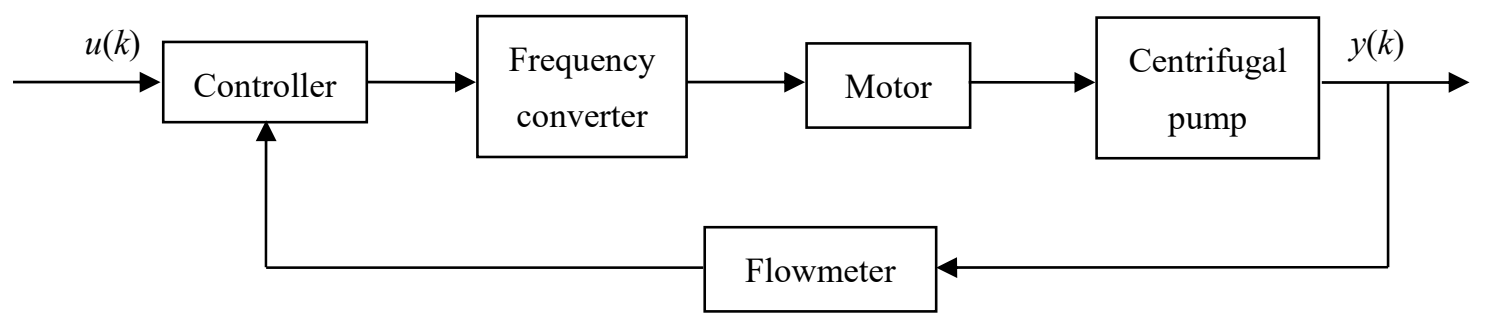

Figure 1. Structure diagram of centrifugal pump flow control system.

\subsection{Frequency Converter Mathematical Model}

The speed regulation of AC motors mainly depends on the frequency converter to change the frequency of the input current of the motor. The control flow is shown in Figure 2. 


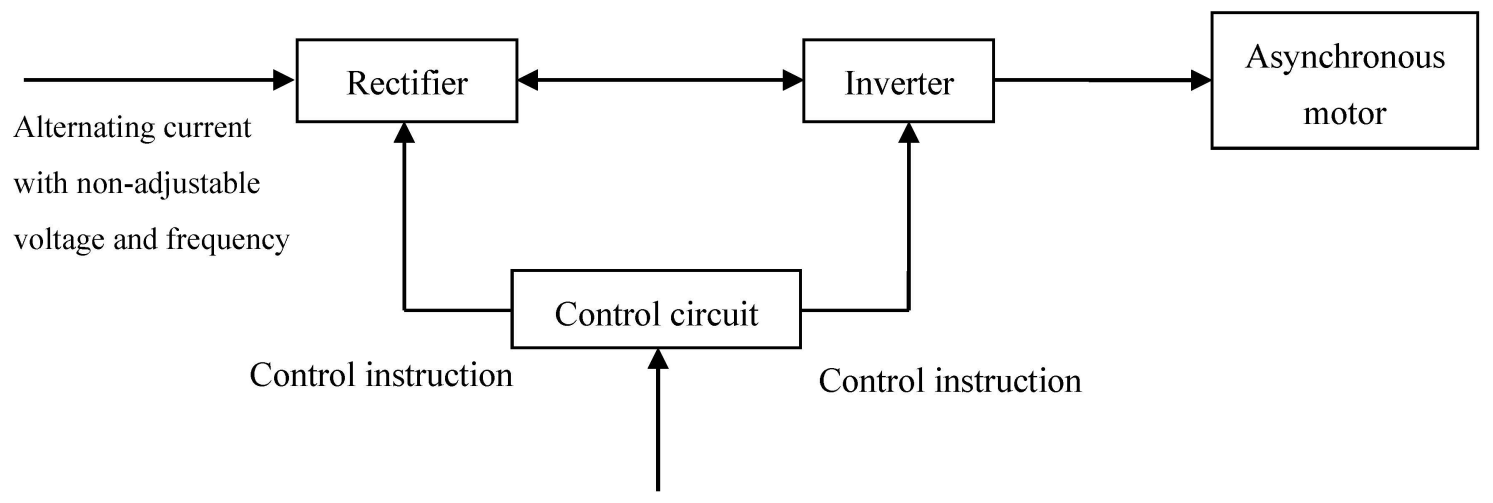

Operating instructions

Figure 2. Flow chart of frequency converter control.

Since the time constant of the frequency converter is much smaller than the time constant of the asynchronous motor, the dynamic response of the frequency converter can be ignored, and the mathematical model can be simplified [7]. The relationship between the input voltage $u_{c}$ and the input current frequency $f_{1}$ of the frequency converter is as follows:

$$
f_{1}=k_{\text {int }} \times u_{c} .
$$

In Equation (1), $f_{1}$ is the power frequency, $k_{\text {int }}$ is the voltage frequency coefficient, and $u_{c}$ is the input voltage of the frequency converter.

The value of $u_{c}$ is $[0-10] \mathrm{V}$, the value of $f_{1}$ is $[0-50] \mathrm{Hz}$, and $k_{\text {int }}=5 \mathrm{~Hz} / \mathrm{V}$ [10].

The relationship between the induced electromotive force $E_{1}$ and stator phase voltage $u_{1}$ in a steady state is [11]:

$$
u_{1}=E_{1}+I_{1}\left(R_{1}+j 2 \pi f_{1} L_{1}\right) .
$$

In Equation (2), $I_{1}$ is the stator current, $L_{1}$ is leakage inductance of each phase of stator winding, and $R_{1}$ is the stator winding resistance.

When the motor works at a low frequency, the torque of the motor decreases greatly, so the compensation voltage $u_{2}$ is required, and its relation formula is [12]:

$$
u_{1}=\frac{220-u_{2}}{50} \times k_{\text {int }} \times u_{c}+u_{2} .
$$

In general, $u_{2}$ is small and can be ignored. The above formula can be changed into:

$$
u_{1}=k_{\text {int }} \times k_{f} \times u_{c} .
$$

In Equation (4), $k_{f}$ is the conversion factor.

According to the above formula, the system control model of the frequency converter is a proportional link, namely:

$$
G_{1}(s)=\frac{u_{1}(s)}{u_{c}(s)}=k_{\text {int }} \times k_{f}=22 .
$$

\subsection{Motor Mathematical Model}

Asynchronous motors generally have two inputs: voltage and frequency. In the process of variable frequency speed control, the two inputs need to be adjusted at the same time. The output of the asynchronous motor generally includes the motor speed and magnetic flux. The magnitude of magnetic flux determines the motor's torque, which is a very important parameter in the motor model. 
The motor model system is relatively complex and integrates high-order, nonlinear and strong coupling. In order to reduce the calculation difficulty, the influences of magnetic circuit saturation, core loss, space harmonics and temperature change are ignored. According to relevant knowledge, the motor model is simplified as an electrical link connected in a series with a mechanical link [13]. The transfer function of the motor system is:

$$
G_{2}(s)=\frac{k_{L}}{\left(T_{L} s+1\right)\left(T_{S} s+1\right)} .
$$

In Equation (6), $T_{L}$ is the mechanical time constant of the motor, $T_{S}$ is the electrical time constant of the motor, and $k_{L}$ is the motor gain factor.

In this paper, $T_{L}=0.012 \mathrm{~s}, T_{S}=0.002 \mathrm{~s}, k_{L}=1$.

\subsection{Centrifugal Pump Mathematical Model}

The centrifugal pump is a kind of pump that relies on a centrifugal force generated by a motor to drive the impeller in order to transfer liquid. Since the working state of the centrifugal pump is very complicated, the following assumptions are made when describing the centrifugal pump mathematical model [11]:

(1) The pulsation of the centrifugal pump is ignored;

(2) The leakage state of the centrifugal pump is laminar flow;

(3) The dynamic process of the centrifugal pump is neglected.

Assuming that leakage is neglected, the actual output flow of the centrifugal pump is equal to the theoretical output flow. The speed formula of the asynchronous motor is as follows:

$$
n=60 f_{1}\left(1-s_{1}\right) / P_{n}
$$

In Equation (7), $f_{1}$ is the power frequency of the asynchronous motor, $s_{1}$ is the slip rate, and $P_{n}$ is the pole logarithm of the asynchronous motor.

Since the output flow rate $Q_{1}$ of the centrifugal pump is proportional to the rotating speed [14], the transfer function of the centrifugal pump mathematical model is as follows:

$$
G_{3}(s)=\frac{Q_{1}(s)}{n_{1}(s)}=k .
$$

\subsection{Control System Mathematical Model}

Combining Equations (5), (6) and (8), and setting $k=1$ in the centrifugal pump mathematical model, it can be seen that the mathematical model of the flow control system is as follows:

$$
G(s)=\frac{k \times k_{L} \times k_{\mathrm{int}} \times k_{f}}{\left(T_{L} s+1\right)\left(T_{S} s+1\right)}=\frac{2,200,000}{2.4 s^{2}+1400 s+100,000}
$$

Since the system is regulated by unit feedback $H(s)$, the closed-loop transfer function of the system is:

$$
G_{k}(s)=\frac{G(s)}{1+G(s) H(s)}=\frac{2200,000}{2.4 s^{2}+1400 s+2,300,000} .
$$

In Equation (10), $H(s)=1$.

\section{Flow Adaptive Control Method for Centrifugal Pump}

\subsection{Control Scheme}

Adaptation is an inherent feature of organisms, which means that when the living environment changes, organisms can change their habits to adapt to environmental changes. Adaptive control is a theory and method of controller design that applies the adaptive characteristics of organisms to controller design. By refining this adaptive feature of biology, 
the controller of the conventional feedback system is used as the inner loop, and then the adaptive outer loop is designed. The adaptive control system is shown in Figure 3 [15].

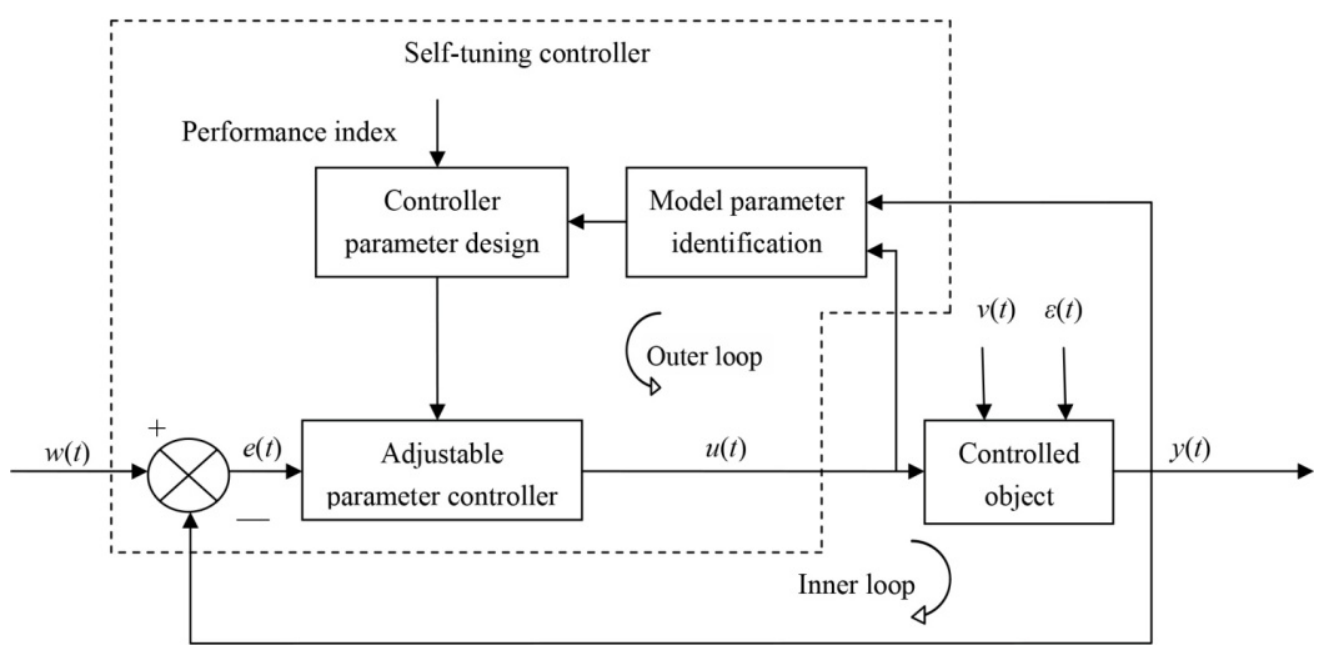

Figure 3. Structure diagram of adaptive control system.

It can be seen from Figure 3 that the adaptive control system is composed of model parameter identification, a controller parameter design, an adjustable parameter controller, a controlled object and a detection device. The actual controlled object is uncertain, so the adaptive control system uses a controller design model with uncertain parameters to describe the controlled object. An adaptive controller with adjustable parameters is designed as the inner loop of the adaptive controller. The outer loop is an adaptive identification control loop for the controller parameter design. The main function of the outer loop is to measure many kinds of information, such as the external interference, input $u(t)$, the output $y(t)$ and the tracking error $e(t)$ between the reference input $w(t)$, and the actual output of the controlled object in real time without interruption, so as to master the environment of the controlled object and its own instability and uncertainty. Based on the obtained object information, according to a certain adaptive theory design method, the control decision is made to update the structure, parameters, or control function of the inner loop controller. Thus, in a sense, optimal or suboptimal control is realized, and a certain desired control target is achieved.

In order to reduce the regulation times of parameters, the self-tuning PID controller is selected to optimize the centrifugal pump flow control system. Its main feature is that the PID controller is used as the parameter adjustable controller in the control system. The self-tuning control theory is adopted to perform self-tuning control on the entire system, reducing the number of adjustments. At the same time, the required parameters can be modified in real-time according to changes to the object characteristics, thus enhancing the stability and robustness of the system and realizing the adaptive control of the control object.

The self-tuning PID controller structure is mainly composed of four parts: the controlled object, least square recursive parameter estimation [16,17], on-line design of controller parameters and an adjustable parameter PID regulator $[18,19]$. The control structure is shown in Figure 4. 


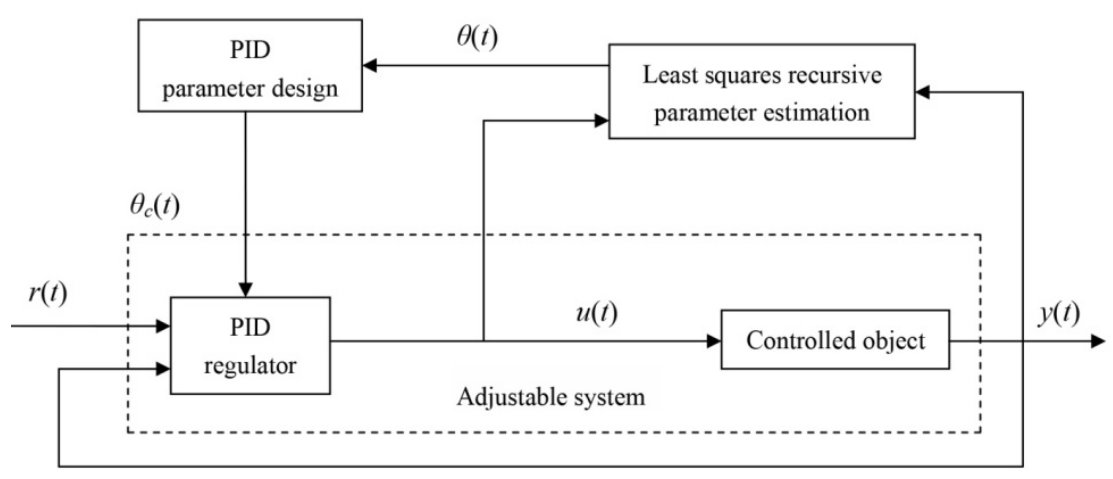

Figure 4. Structure diagram of self-tuning PID control system.

\subsection{Description of Controlled Object Model}

The controlled object of self-tuning control can be described by a discrete-time stochastic linear model. The recursive least squares algorithm is used to calculate the model. The discrete-time stochastic linear model is [20]:

$$
A\left(z^{-1}\right) y(k)=B\left(z^{-1}\right) u(k-d)+\varepsilon(k) .
$$

In Equation (11), $u(k)$ is the input variable of the controlled object, $y(k)$ is the output variable of the controlled object, $\varepsilon(k)$ is white noise, and $d$ is the number of output delays of the controlled object.

Setting the sampling period $T_{S}=0.01 \mathrm{~s}$, the centrifugal pump control system $G_{k}(s)$ is discretized by the backward difference method to obtain:

$$
G_{k}\left(z^{-1}\right)=\frac{1.007 z^{-1}+0.05569}{z^{-2}+0.1079 z^{-1}+0.002928} .
$$

To set the output delay number $d=1$, the random linear model disturbed by white noise is:

$$
\left(1+36.85 z^{-1}+341.53 z^{-2}\right) y(k)=\left(19.02+343.92 z^{-1}\right) u(k-1)+\varepsilon(k) .
$$

In Equation (13), $\varepsilon(k)$ is constant interference.

\subsection{Real-time Parameter Estimation Algorithm}

The forgetting factor recursive least squares method is used to estimate the real-time parameters of the centrifugal pump control system [21]. Equation (11) is equivalently converted into:

$$
y(k)=G\left(z^{-1}\right) u(k)+H\left(z^{-1}\right) \varepsilon(k) .
$$

In Equation (14), $G\left(z^{-1}\right)=B\left(z^{-1}\right) / A\left(z^{-1}\right)$ is the system model, and $H\left(z^{-1}\right)=1 / A\left(z^{-1}\right)$ is the noise model. It can be expressed as:

$$
y(k)=\varphi^{T}(k) \theta+\varepsilon(k) .
$$

In Equation (15), parameter vector $\theta=\left[a_{1}, a_{2}, \cdots a_{n_{a}}, b_{1}, b_{2}, \cdots b_{n_{b}}\right]^{T}$.

The data information vector $\varphi(k)$ is:

$$
\varphi(k)=\left[-y(k-1),-y(k-2), \cdots-y\left(k-n_{a}\right), u(t-d-1), u(t-d-2), \cdots u\left(t-d-n_{b}\right)\right]^{T}
$$

The performance index is as follows:

$$
J=\sum_{K=1}^{L} \lambda^{L-K}\left[y(k)-\varphi^{T}(k) \hat{\theta}\right]^{2}
$$


In Equation (17), $\lambda$ is the forgetting factor $(0<\lambda \leq 1)$.

The algorithm expression of forgetting factor recursive least squares parameter estimation is as follows [22]:

$$
\left\{\begin{array}{c}
\hat{\theta}(k)=\hat{\theta}(k-1)+K(k)\left[y(k)-\varphi^{T}(k) \hat{\theta}(k-1)\right] \\
K(k)=P(k-1) \varphi(k)\left[\lambda+\varphi^{T}(k) P(k-1) \varphi(k)\right]^{-1} . \\
P(k)=\frac{1}{\lambda}\left[1-K(k) \varphi^{T}(k) P(k-1)\right]
\end{array} .\right.
$$

PID control is adopted for the system. In order to eliminate constant disturbance, the controller must have an integral link [23]. The corresponding PID controller can be expressed as:

$$
F_{1}\left(z^{-1}\right) u(k)=R\left(z^{-1}\right) y_{r}(k)-G\left(z^{-1}\right) y(k) .
$$

In Equation (19), $F_{1}\left(z^{-1}\right)=F\left(z^{-1}\right) \Delta$.

Further:

$$
\left\{\begin{array}{c}
F\left(z^{-1}\right)=1+f_{1}\left(z^{-1}\right)+\cdots+f_{n_{f}}\left(z^{-n_{f}}\right) \\
G\left(z^{-1}\right)=g_{0}+g_{1}\left(z^{-1}\right)+g_{2}\left(z^{-2}\right) \\
R\left(z^{-1}\right)=G(1)=g_{0}+g_{1}+g_{2}
\end{array} .\right.
$$

Equation (20) is arranged so that the closed-loop characteristic polynomial is the denominator polynomial of the desired transfer function, thus obtaining:

$$
A F+z^{-d} B G=A_{m} .
$$

\section{System Simulation and Analysis}

\subsection{Parameter Determination}

The standard form of the second-order system is:

$$
G(s)=\frac{\omega_{n}^{2}}{s^{2}+2 \xi \omega_{n} s+\omega_{n}^{2}} .
$$

The following results can be obtained by calculation: $\xi=0.2979$, and the optimal damping ratio is adjusted to $\xi_{e}=0.707$.

After adjustment, the expected transfer function is:

$$
G_{e}(s)=\frac{2,200,000}{2 \cdot 4 s^{2}+3322 s+2,300,000} .
$$

The difference equation is:

$$
\left(1-1624.92 z^{-1}+1026483.27 z^{-2}\right) y(k)=\left(-197.29+980,496.82 z^{-1}\right) u(k)
$$

The initial value $P_{0}=10^{6} I, \hat{\theta}(0)=0.001$ and forgetting factor $\lambda=0.97$ of the PID controller are set to optimize the original system.

The parameter optimization process of the self-tuning PID control algorithm is as follows:

(1) The initial data is inputted, and the initial values $\hat{\theta}(0), P(0)$ and the forgetting factor $\lambda$ are set;

(2) The current actual output of the acquisition system is $y(k)$;

(3) The real-time parameter $\hat{\theta}$ of the control target is estimated by the recursive least squares method with the forgetting factor through model parameter identification;

(4) Solving $F$ and $G$ in Equation (20), taking $F_{1}=\Delta F$ and $R=G(1)$;

(5) Calculate the control quantity $u(k)$ and regulate the system through Equation (19);

(6) Return to step (2) and continue the loop $(k \rightarrow k+1)$.

The object parameter estimation results are shown in Figure 5: 


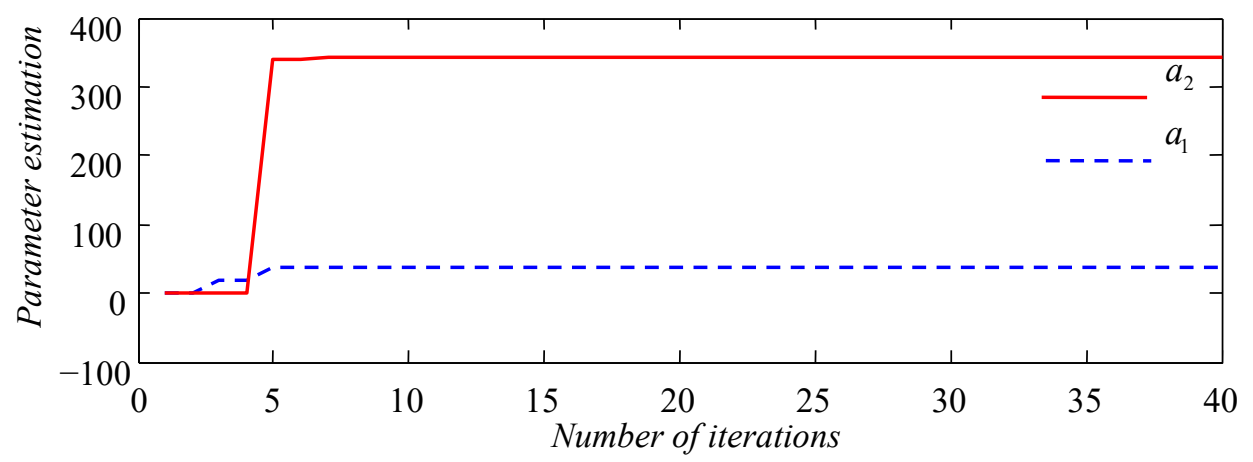

(a) Parameter estimation

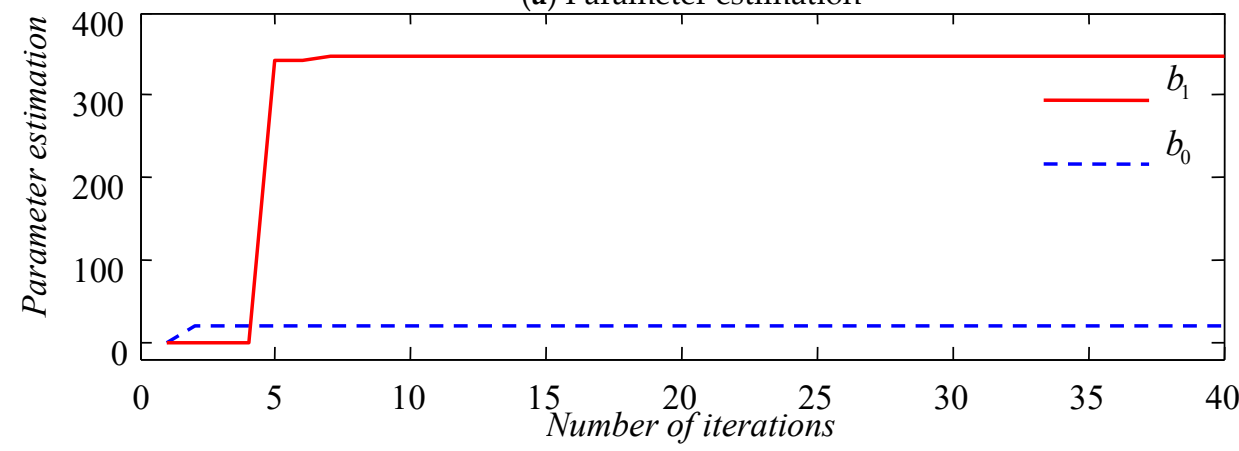

(b) Parameter estimation

Figure 5. Object parameter estimation results.

As can be seen from Figure 5, the object parameters are estimated to be $a_{1}=36.85$, $a_{2}=341.53, b_{0}=19.02$, and $b_{1}=343.92$.

Then: $A_{m}\left(z^{-1}\right)=1-1624.92 z^{-1}+1,026,483.27 z^{-2}$.

The optimized difference equation is:

$$
\left(1+37 z^{-1}+344.3 z^{-2}\right) y(k)=\left(19.02+346.7 z^{-1}\right) u(k) .
$$

The optimized system transfer function is:

$$
G_{r}(s)=\frac{201.1 s+171,400}{s^{2}+584.2 s+179,200} .
$$

\subsection{System Simulation and Analysis}

The input signal is set as a unit step signal, and the original system $G_{k}(s)$ and the system $G_{r}(s)$ after adding self-tuning PID control are simulated, respectively. The system response curves are shown in Figures 6 and 7, respectively.

The system transient response indicators before and after optimization are extracted from Figures 6 and 7, as shown in Table 1.

Table 1. Transient response indexes of original system and optimized system.

\begin{tabular}{cccc}
\hline $\begin{array}{c}\text { Transient Response } \\
\text { Index }\end{array}$ & Original System & Optimized System & $\begin{array}{c}\text { Performance Change } \\
\text { (\%) }\end{array}$ \\
\hline Rise time/s & 0.00135 & 0.00425 & -214.81 \\
Peak time/s & 0.0033 & 0.0067 & -103.03 \\
Adjusting time/s & 0.0187 & 0.0156 & 16.58 \\
Maximum overshoot & 0.3752 & 0.0604 & 83.90 \\
\hline
\end{tabular}




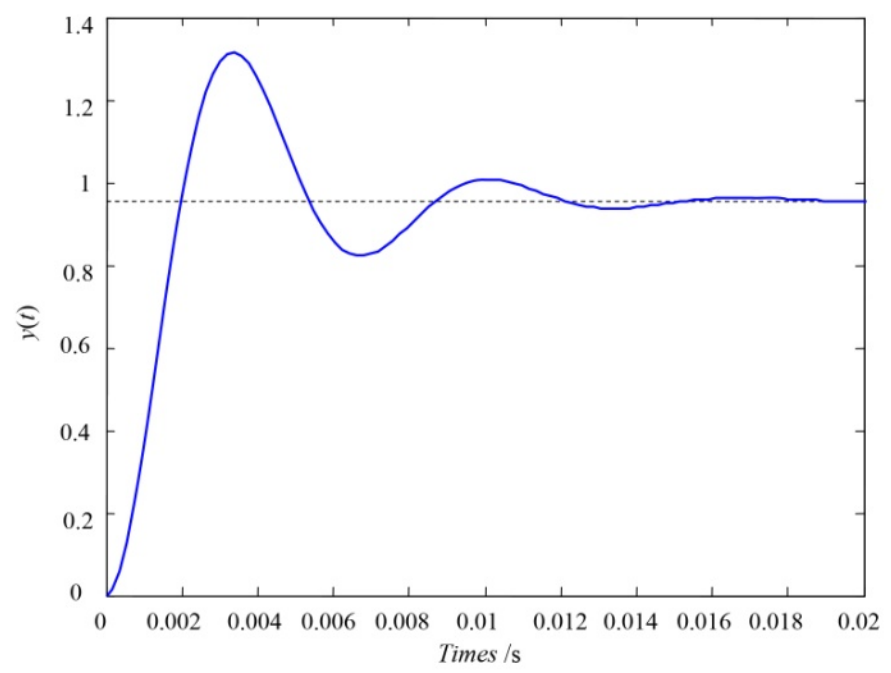

Figure 6. Step response curve of original system.

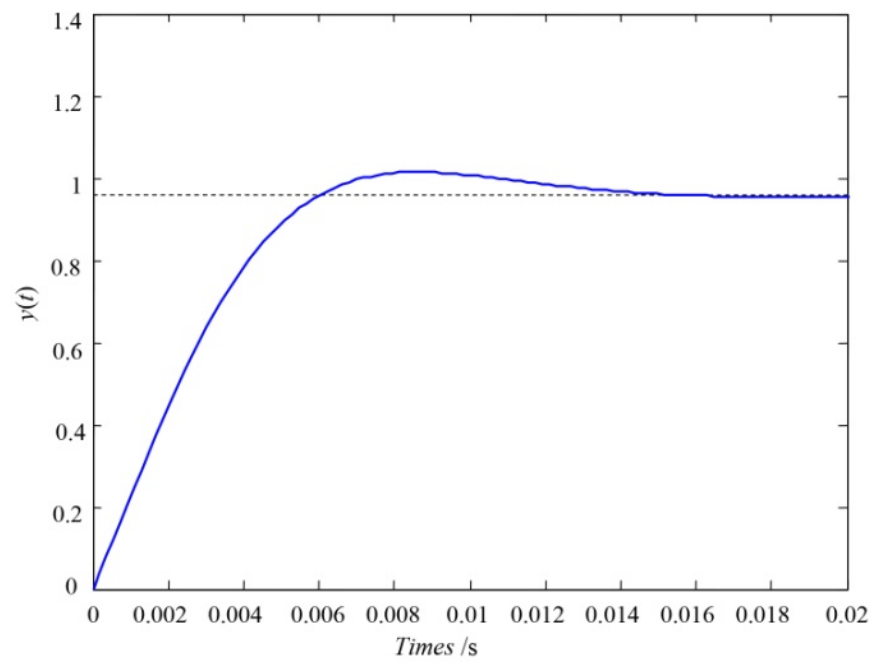

Figure 7. Step response curve of system after adding self-tuning PID control.

According to the data analysis in Table 1, the rise time and peak time of the centrifugal pump flow control system after adding the self-tuning PID controller are both large. When parameter identification and PID control are added, the control process of the system obviously increases. The control time of the system also increases. Although the optimized control time increases, the adjustment time to a steady state after system optimization is shortened by $16.58 \%$, which improves the rapidity of the system transient response. The optimized output response curve of the system is relatively gentle, the maximum overshoot is reduced by $83.90 \%$, and the stability of the system is greatly improved.

\section{Experimental Analysis of Centrifugal Pump Flow Adaptive Control System}

\subsection{Test Platform Construction}

In order to verify the accuracy of the self-tuning PID control algorithm, the NGL002 centrifugal pump comprehensive test device is selected to build a centrifugal pump flow adaptive control system test platform. The test device and test platform are shown in Figures 8 and 9 , respectively. 


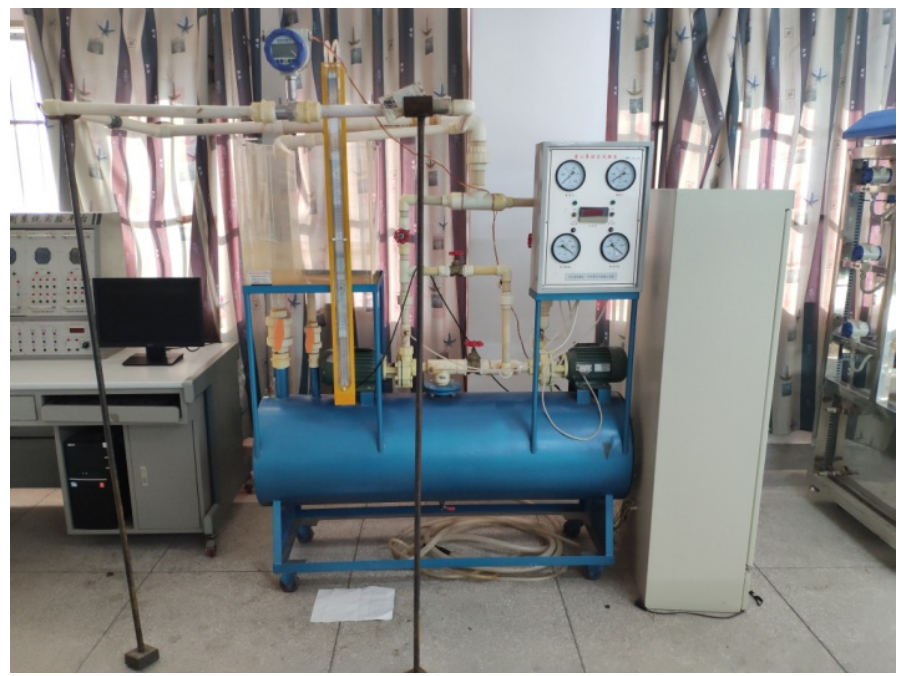

Figure 8. Centrifugal pump comprehensive test device.

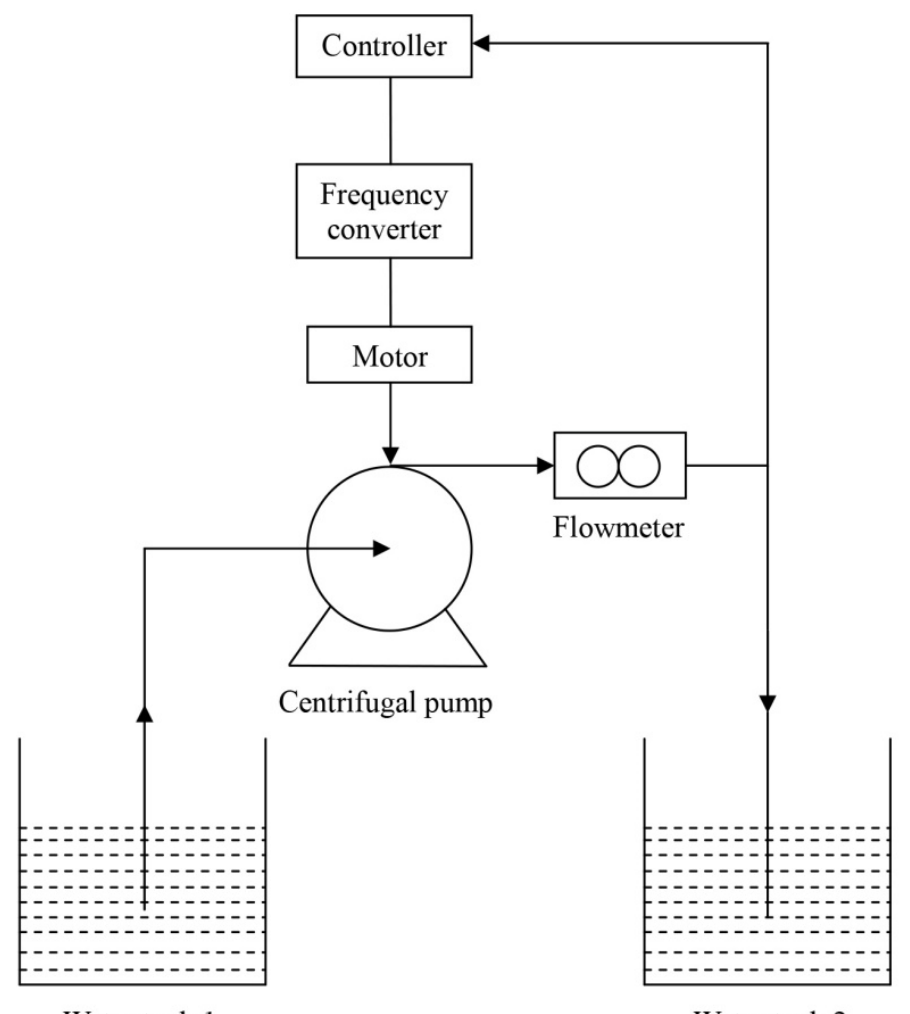

Water tank 1

Water tank 2

Figure 9. Test platform of centrifugal pump flow adaptive control system.

It can be seen from Figure 9 that the centrifugal pump draws water from the first water tank, and that the water flows through the flowmeter to be injected into the second water tank. The flowmeter feeds the flow signal back to the controller. The controller controls the motor speed through the frequency converter, and then controls the output flow of the centrifugal pump.

\subsection{Data Acquisition and Analysis of Constant Frequency Flow}

The rated flow rate of the centrifugal pump used in the test is $Q_{e}=5 \mathrm{~m}^{3} / \mathrm{h}$, and the electrical frequency is $f=50 \mathrm{~Hz}$. The working frequency of the frequency converter is set to $f_{1}=45.8 \mathrm{~Hz}$, and data are collected every $10 \mathrm{~s}$ for 10 times in total. The data acquisition results are shown in Table 2 . 
Table 2. Test results collection.

\begin{tabular}{cccc}
\hline Times/s & Theoretical Flow $\mathbf{A} /\left(\mathbf{m}^{3} / \mathbf{h}\right)$ & Measured Flow $\mathbf{B} /\left(\mathbf{m}^{3} / \mathbf{h}\right)$ & Error Rate $\mathbf{( \% )}$ \\
\hline 10 & 3.954 & 3.852 & 2.580 \\
20 & 3.951 & 3.848 & 2.607 \\
30 & 3.958 & 3.853 & 2.653 \\
40 & 3.948 & 3.845 & 2.609 \\
50 & 3.953 & 3.847 & 2.682 \\
60 & 3.956 & 3.851 & 2.654 \\
70 & 3.952 & 3.849 & 2.606 \\
80 & 3.957 & 3.854 & 2.603 \\
90 & 3.954 & 3.852 & 2.580 \\
100 & 3.949 & 3.844 & 2.659 \\
\hline
\end{tabular}

Curve fitting is performed according to the data in Table 2 [24]. The curves of theoretical flow and actual collected flow can be obtained at a constant frequency, as shown in Figure 10.

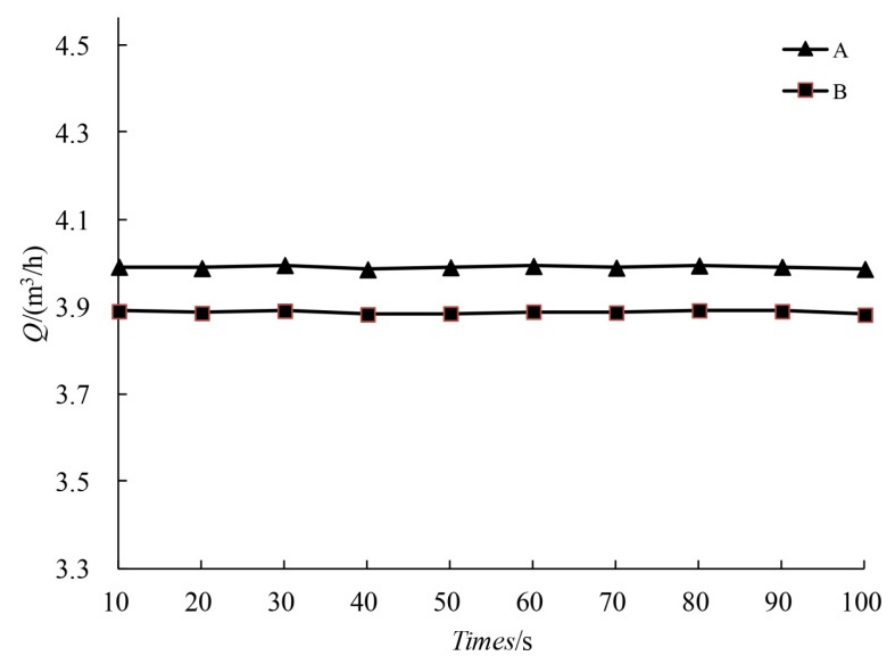

Figure 10. Constant frequency flow curve fitting.

From Table 2 and Figure 10, it can be seen that the theoretical flow rates of the pump are always greater than the actual flow rates at a constant frequency. The main reason for this is that the pump has a certain amount of leakage during operation. With the increase in acquisition time, the theoretical flow rate of the pump is always maintained at about $3.95 \mathrm{~m}^{3} / \mathrm{h}$, and the variation range is less than $0.25 \%$. The actual flow rate of the pump is basically kept around 3.95, and the change range is less than $0.26 \%$. Therefore, under the condition of constant frequency, the adaptive PID regulator can effectively adjust the system parameters and improve the stability of the pump. The error between the actual flow curve and the theoretical flow curve of the pump is not large, and the accuracy is relatively high, which is basically controlled within $3 \%$.

\subsection{Data Acquisition and Analysis of Variable Frequency Flow}

The theoretical flow of the centrifugal pump is $Q_{l}=Q_{e} \bullet f_{1} / f$, and the theoretical flow of the pump is directly proportional to the operating frequency of the frequency converter. The output frequency of the frequency converter and the actual flow rate of the pump can be acquired through experiments. The upper limit value of the frequency converter's working frequency is set to $48 \mathrm{~Hz}$, and the lower limit value is set to $30 \mathrm{~Hz}$. The data acquisition results are shown in Table 3. 
Table 3. Test results collection.

\begin{tabular}{cccc}
\hline Frequency $f_{1} / \mathbf{H z}$ & Theoretical Flow $A /\left(\mathbf{m}^{3} / \mathbf{h}\right)$ & Measured Flow $\mathbf{B} /\left(\mathbf{m}^{3} / \mathbf{h}\right)$ & Error Rate $(\%)$ \\
\hline 30 & 2.458 & 2.428 & 1.221 \\
32 & 2.603 & 2.586 & 0.653 \\
34 & 2.865 & 2.822 & 1.501 \\
36 & 3.002 & 2.954 & 1.599 \\
38 & 3.223 & 3.198 & 0.776 \\
40 & 3.408 & 3.357 & 1.496 \\
42 & 3.672 & 3.644 & 0.763 \\
44 & 3.856 & 3.797 & 1.530 \\
46 & 4.106 & 4.102 & 0.097 \\
48 & 4.603 & 4.589 & 0.304 \\
\hline
\end{tabular}

Curve fitting is performed according to the data in Table 3. The curves of the theoretical flow and actual collected flow can be obtained at a variable frequency, as shown in Figure 11.

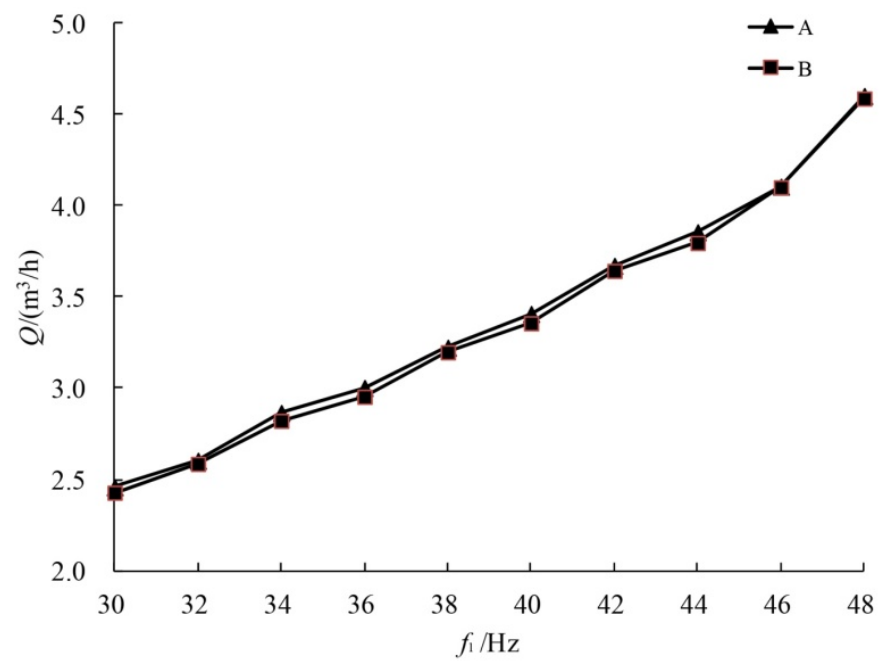

Figure 11. Variable frequency flow curve fitting.

As can be seen from Table 3 and Figure 11, the upper and lower limits of the working frequency of the constant frequency are set to achieve accurate control of the pump flow. As the frequency increases, the theoretical flow and actual flow of the pump both increase in direct proportion. This shows that under the condition of frequency conversion, the designed adaptive control method can effectively improve the working efficiency of the pump, and the control quality is good. With the change in the working frequency of the frequency converter, the actual flow rates of the pump are close to the theoretical flow rates. The actual flow is always kept near the preset flow, and the error is controlled within $2 \%$. Therefore, the optimized centrifugal pump flow control system has better real-time followability.

\section{Conclusions}

(1) By analyzing the components of a frequency converter, motor, and centrifugal pump, the frequency converter and centrifugal pump were simplified as a proportional link. The motor was simplified as an electrical link connected in a series with a mechanical link, and the mathematical model of the centrifugal pump adaptive control system was established.

(2) A self-tuning PID controller based on the adaptive control method was constructed, a discrete-time stochastic linear model was used to describe the controlled object model, and the forgetting factor recursive least squares method was used to estimate the pa- 
rameters in real-time. The simulation results showed that the dynamic characteristics of the optimized system were significantly improved, and the rapidity and stability of the transient response of the system were both improved.

(3) The test platform was built based on the NGL002 centrifugal pump comprehensive test device, and a good control effect was obtained. After optimization, the centrifugal pump flow control system had strong robustness and had better real-time followability. The accuracy of the self-tuning PID control algorithm was further verified.

(4) In order to improve the robustness and stability of the centrifugal pump, the mathematical model of the control system was obtained on the basis of deducing the mathematical model of the frequency converter, motor and centrifugal pump. The adaptive control algorithm was used to optimize the design of the system. The least squares recursive algorithm was used to estimate the parameters, and the self-tuning PID controller was added to adjust the parameters of the centrifugal pump control system. The experimental results showed that the optimized centrifugal pump control system shortened the adjustment time, reduced the maximum overshoot, and the system's rapidity and stability were significantly improved. Based on the test platform of the centrifugal pump flow adaptive control system, the flow data of a fixed frequency and variable frequency were collected. The collected data showed that the error between the actual flow curve and the theoretical flow curve of the pump was small, which verified the accuracy of the self-tuning PID control algorithm. It could be seen that based on the adaptive control algorithm, the performance of each index of the centrifugal pump flow control system could be improved, and the stability and robustness of the system could also be improved. This has certain guiding significance for the ability of centrifugal pumps to effectively cope with various complicated working conditions and to improve work efficiency during the operation process.

Author Contributions: Conceptualization, Y.W.; methodology, Z.H.; software, H.Z.; validation, Y.W., H.Z. and X.N.; formal analysis, Z.H.; investigation, X.N.; resources, Z.H.; data curation, X.N.; writing_-original draft preparation, Y.W.; writing—review and editing, Y.W.; visualization, Y.W.; supervision, Y.W.; project administration, Y.W.; funding acquisition, Y.W. All authors have read and agreed to the published version of the manuscript.

Funding: This research was funded by the Anhui Province University Discipline (Professional) Top Talent Academic Funding Project (No. gxbjZD2021076). This project was funded by the National College Student Innovation and Entrepreneurship Training Program Project (No. 202110380020). This project was funded by the Key Project of Natural Science Research in Colleges and Universities of Anhui Province (No. KJ2017A450). This project was funded by the Key Project of Natural Science Foundation of Chaohu University (No. XLZ-201902).

Institutional Review Board Statement: Not applicable.

Informed Consent Statement: Not applicable.

Data Availability Statement: The study did not report any data.

Conflicts of Interest: The authors declare that there is no conflict of interest regarding the publication of this paper.

\section{References}

1. Wang, X.; Kuang, K.; Wu, Z.; Yang, J. Numerical simulation of axial vortex in a centrifugal pump as turbine with S-blade impeller. Processes 2020, 8, 1192. [CrossRef]

2. Gülich, J.F. Centrifugal Pumps; Springer: Heidelberg, Germany, 2010.

3. Lobanoff, V.S.; Ross, R.R. Centrifugal Pumps: Design and Application; Gulf Publishing Company: Houston, TX, USA, 1992.

4. Gevorkov, L.; Rassõlkin, A.; Kallaste, A.; Vaimann, T. Simulink based model for flow control of a centrifugal pumping system. In Proceedings of the International Workshop on Electric Drives: Optimization in Control of Electric Drives, Moscow, Russia, 31 January-2 February 2018; pp. 1-4.

5. Suh, S.H.; Kim, K.W.; Kim, H.H.; Yoon, I.S.; Cho, M.T. A Study on energy saving rate for variable speed condition of multistage centrifugal pump. J. Therm. Sci. 2015, 24, 566-573. [CrossRef] 
6. Mingxin, C.; Lingfeng, T.; Jian, H. Research on flow control system of pump based on fuzzy PID. J. Xinxiang Univ. 2014, 31, 36-40. (in Chinese).

7. Lu, J.; Chen, M. Reach on the Ho control system of the centrifugal pumps. J. Tongren Univ. 2018, 20, 68-75. (in Chinese).

8. Xie, S.; Chai, T. A kind of fuzzy PID controller with parameter adaption. Inf. Control. 1998, 27, 255-259. (in Chinese).

9. Perissinotto, R.M.; Verde, W.M.; Perles, C.E.; Biazussi, J.L.; de Castro, M.S.; Bannwart, A.C. Experimental analysis on the behavior of water drops dispersed in oil within a centrifugal pump impeller. Exp. Therm. Fluid Sci. 2019, 112, 1-18. [CrossRef]

10. Hu, J. Research of Wet Desulfurization Dust Control System. Master's Thesis, Anhui Polytechnic University, Wuhu, China, 2014. (in Chinese).

11. Gang, C. The Research of The Method on The Frequency Conversion Energy-Conservation of the Hydraulic Apparatus Controls. Master's Thesis, Guizhou University, Guiyang, China, 2010. (in Chinese).

12. Zeng, G.; Qian, M. Frequency conversion driving technology of AC asynchronous motor. Electr. Abstr. 2014, 5, 13-17. (in Chinese).

13. Jun, W. Research on low frequency torque insufficiency of frequency converter. China Sci. Technol. Inf. 2015, 2, 183-184. (in Chinese).

14. Zhao, X.S.; Hu, J.; Shi, P.C. Hydromechanics research of pump flow control system based on BP neural network PID. Appl. Mech. Mater. 2013, 327, 222-226. [CrossRef]

15. Wen, M. Analysis of flow adjustment method of centrifugal pump. China Pet. Chem. Stand. Qual. 2012, z2, 117. (in Chinese).

16. Jiang, J.; Zhang, Y. A revisit to block and recursive least squares for parameter estimation. Comput. Electr. Eng. 2004, 30, 403-416. [CrossRef]

17. Ding, F.; Wang, X.; Chen, Q.; Xiao, Y. Recursive least squares parameter estimation for a class of output nonlinear systems based on the model decomposition. Circuits Syst. Signal Process. 2016, 35, 3323-3338. [CrossRef]

18. Alexandrov, A.; Palenov, M. Self-tuning PID-I controller. Autom. Remote Control 2011, 44, 3635-3640. [CrossRef]

19. Makarem, S.; Delibas, B.; Koc, B. Data-driven tuning of PID controlled piezoelectric ultrasonic motor. Actuators 2021, 10, 148. [CrossRef]

20. Xie, X.; Ding, F. Adaptive Control System; Tsinghua University Press: Beijing, China, 2002. (in Chinese)

21. Paleologu, C.; Benesty, J.; Ciochina, S. A robust variable forgetting factor recursive least-squares algorithm for system identification. IEEE Signal Process. Lett. 2008, 15, 597-600. [CrossRef]

22. Pang, Z.; Cui, H. System Identification and Adaptive Control Matlab Simulation; Beihang University Press: Beijing, China, 2013. (in Chinese)

23. Luo, H.; Mao, Y.; Zhang, J. Design and simulation of a parameter adaptive fuzzy PID controller. Autom. Instrum. 2001, 3, 10-12. (in Chinese).

24. Hu, M.; Feng, J.; Zheng, J. An additional branch free algebraic B-spline curve fitting method. Vis. Comput. 2010, 26, 801-811. [CrossRef] 